\section{ASSESSMENT OF VALVE HAEMODYNAMICS, REVERSE VENTRICULAR REMODELLING AND MYOCARDIAL FIBROSIS FOLLOWING TRANSCATHETER AORTIC VALVE IMPLANTATION COMPARED TO SURGICAL AORTIC VALVE REPLACEMENT. A CARDIOVASCULAR MAGNETIC RESONANCE STUDY}

doi:10.1136/heartjnl-2012-301877b.42

${ }^{1} \mathrm{~T}$ A Fairbairn, ${ }^{*} \mathrm{C}$ D Steadman, ${ }^{1} \mathrm{~A} N$ Mather, ${ }^{1} \mathrm{M}$ Motwani, ${ }^{3} \mathrm{D}$ J Blackman, ${ }^{1} \mathrm{~S}$ Plein, ${ }^{2} \mathrm{G} P$ McCann, 'J P Greenwood. ${ }^{1}$ University of Leeds, Leeds, UK; ${ }^{2}$ University of Leicester, UK; ${ }^{3}$ Leeds General Infirmary, Leeds, UK

Introduction One-year survival post transcatheter aortic valve implantation (TAVI) is non-inferior to surgical aortic valve replacement (SAVR) in patients with severe aortic stenosis (AS) and high operative risk. The effects of TAVI on valve haemodynamics and subsequent ventricular reverse remodelling relative to SAVR are less certain, and the impact of myocardial fibrosis (MF) is unknown. These would be expected to impact on longer-term outcome. Our aim was to use cardiovascular magnetic resonance (CMR) imaging to assess the 6-month post-operative aortic valve haemodynamics, reverse ventricular remodelling, and myocardial fibrosis changes following TAVI compared to SAVR. Secondary aims were to identify predictors of impaired left ventricular reverse remodelling and to establish the importance of pre-operative myocardial fibrosis on clinical outcomes.

Methods 77 high-risk AS patients referred for TAVI or SAVR were prospectively recruited. 50 patients (25 TAVI, 25 SAVR) completed baseline and 6-month post-operative 1.5 Tesla CMR scans. Multislice, multi-phase cine imaging was performed to cover the entire left ventricle. Phase contrast (velocity encoded) imaging was used to quantify aortic mean gradient and \% regurgitation. Late gadolinium enhancement was performed $10 \mathrm{~min}$ after the administration of $0.2 \mathrm{mmol} / \mathrm{kg}$ of Gadoteric acid (Doteram, Guerbet, SA, Villepinte). TAVI used the third generation CoreValve revalving system. In the surgical patients $96 \%$ had bioprosthetic valves.

Results Patients were matched for gender, BMI, AS severity and the majority of comorbidities, but not for age ( $80 \pm 6$ vs $73 \pm 7$ years, $\mathrm{p}=0.001)$ or EuroSCORE ( $22 \pm 14$ vs $7 \pm 3, p<0.001)$. Aortic valve mean pressure gradient decreased to a greater degree $(p=0.017)$ 6 months post-TAVI $(50 \pm 16$ vs $21 \pm 8 \mathrm{~mm} \mathrm{Hg}, \mathrm{p}<0.001)$ compared to SAVR $(55 \pm 20$ vs $35 \pm 13 \mathrm{~mm} \mathrm{Hg}, \mathrm{p}<0.001)$. AR was reduced by $8 \%$ in each group, only reaching statistical significance for TAVI $(p=0.003)$. Post-operative ventricular end-systolic volumes (ESVI) and mass improved in both groups $(p<0.05)$, additionally SAVR reduced end-diastolic volumes (EDVI, $\mathrm{p}<0.001)$ and TAVI increased ejection fraction ( $E F, p=0.01$ ), Abstract 042 table 1. Concentric remodelling (mass/EDV: $0.88 \pm 0.2$ vs $0.73 \pm 0.2, \quad \mathrm{p}<0.001$ ) and geometric wall function (thickness and thickening), improved postTAVI $(p<0.001)$ but not post-SAVR ( $p>0.05)$, Abstract 042 figure 1.

Abstract 042 Table 1

\begin{tabular}{|c|c|c|c|c|c|}
\hline & \multicolumn{2}{|l|}{ TAVI } & \multicolumn{2}{|l|}{ SAVR } & \multirow[b]{2}{*}{ p Value $\dagger$} \\
\hline & Baseline & 6 months & Baseline & 6 months & \\
\hline \multicolumn{6}{|l|}{ Function } \\
\hline LVEDVI, ml/m² & $94 \pm 18$ & $90 \pm 20$ & $92 \pm 19$ & $74 \pm 12$ & 0.04 \\
\hline LVESVI, ml/m² & $46 \pm 18$ & $41 \pm 17$ & $44 \pm 22$ & $32 \pm 6$ & 0.19 \\
\hline LVSVI, ml/m² & $48 \pm 10$ & $50 \pm 10$ & $49 \pm 8$ & $42 \pm 7$ & 0.14 \\
\hline LVEF, \% & $52 \pm 12$ & $56 \pm 10$ & $55 \pm 11$ & $57 \pm 8$ & 0.57 \\
\hline LVMI, $g / \mathrm{m}^{2}$ & $82 \pm 20$ & $65 \pm 17$ & $74 \pm 11$ & $59 \pm 8$ & 0.35 \\
\hline LVM/LVEDV, $\mathrm{g} / \mathrm{ml}$ & $0.88 \pm 0.2$ & $0.73 \pm 0.2$ & $0.80 \pm 0.1$ & $0.81 \pm 0.2$ & $<0.001$ \\
\hline \multicolumn{6}{|l|}{ Aortic valve } \\
\hline $\mathrm{MPG}, \mathrm{mm} \mathrm{Hg}$ & $50 \pm 16$ & $21 \pm 8$ & $55 \pm 20$ & $35 \pm 13$ & 0.017 \\
\hline AR fraction, $\%$ & $16 \pm 11$ & $8 \pm 6$ & $18 \pm 7$ & $10 \pm 11$ & 0.46 \\
\hline
\end{tabular}

MF burden and low EF were associated with greater post-operative remodelling in both groups by univariate analysis. EF remained an independent predictor on multivariate analysis $(p<0.001)$ MF showed evidence of regression post-TAVI $(p=0.04)$ but not post-SAVR.
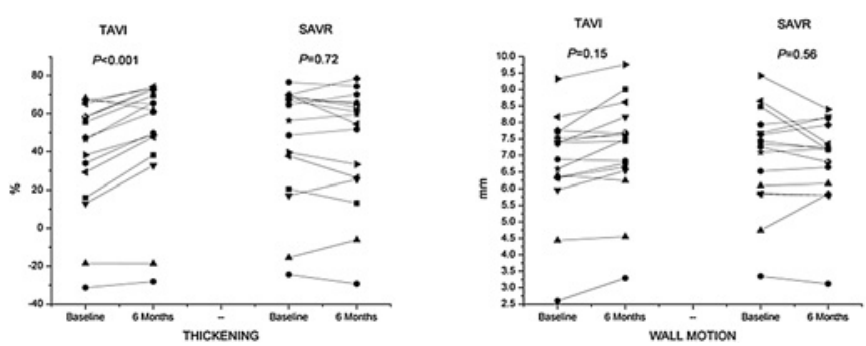

Abstract 042 Figure 1

Conclusion In high-risk AS patients, TAVI compared to SAVR produced a greater improvement in the aortic valve pressure gradient, concentric LV reverse remodelling, geometric wall function and MF. EF rather than MF was a more powerful predictor of this process.

\section{LOW PACING RATE ACHIEVED IN COREVALVE TRANSCATHETER AORTIC VALVE IMPLANTATION (TAVI): COMPARISON OF PACING RATE PRE AND POST NEW DELIVERY CATHETER}

doi:10.1136/heartjnl-2012-301877b.43

M Drury-Smith,* S Lakshmanan, R Giri, M Fayaz, J Cotton, M Bhabra, S Khogali. Heart and Lung Centre, New Cross Hospital, UK

Introduction Permanent pacemaker implantation (PPM) following transcatheter aortic valve implantation (TAVI) is a recognised complication. However, the higher pacing rate post CoreValve TAVI compared to SAVR ( $33 \%$ vs $8 \%$ ) is a cause for concern. Several factors including pre-existing bundle branch block (BBB), larger valve size, post dilatation and low implantation have been shown to independently impact on an increased risk of PPM requirement. Depth of CoreValve implantation below the aortic annulus can result in compression of conduction tissue and heart block and is therefore an important predictor of PPM requirement. A modified delivery catheter (ACCUTRAK) was introduced to address this by providing more controlled release of the prosthesis, preventing low implantation, thereby reducing the pacing rate. We evaluated the pacing rate in our cohort of patients (pts) and the effect of the new Accutrak catheter on the pacing rate.

Methods TAVI was performed in 91 patients, mean age ( 82.9 years) The trans-femoral route (72 patients), the left subclavian route (15 patients) and direct aortic approach (4 patients) was used with a consistently high valve deployment strategy of $3-5 \mathrm{~mm}$ below the aortic annulus. 46 patients had TAVI with pre-Accutrak catheter

\section{Abstract 043 Table 1}

\begin{tabular}{ll}
\hline & Procedural results \\
\hline Mean age (years) & 82.9 \\
Mean logistic Euroscore & 19.3 \\
Success rate (\%) & 99 \\
Mortality rate (\%) & 2.2 \\
Vascular complications (\%) & 5 \\
Stroke rate (\%) & 2.2 \\
Permanent pacemaker rate post TAVI* (\%) & 9.8 \\
\hline
\end{tabular}

*New pacemaker post TAVI within 30 days of procedure. 
and 35 patients with the Accutrak catheter. Procedural outcomes were analysed (Abstract 043 table 1) with detailed evaluation of preTAVI PPM, pre-procedure ECG, annulus size, pre-dilatation balloon size, CoreValve size, and post-dilatation in the two different groups. Results 81 patients of the 91 had no pre-existing PPM. CoreValve prosthesis was deployed high, 3-5 mm, below aortic annulus. Factors previously identified as predictors of PPM requirement post TAVI, were similar in both groups (Abstract 043 table 2). A total of eight patients required a new PPM with a pacing rate post TAVI of 9.8\%. There were 5 PPM implantations in the pre-Accutrak group and 3 in the Accutrak group. In the pre-Accutrak ppm group, 2 patients had sinus rhythm (SR) plus LBBB,1 AF and LBBB,1SR with $\mathrm{RBBB}$ and 1SR. In the Accutrak PPM group (3 patients) 2 had SR with RBBB and 1 with SR and 1st degree heart block. There was no significant difference in the pacing rate between the pre-Accutrak $(10.9 \%)$ and post-Accutrak ( $8.6 \%)$ groups $(p=1.0)$ in this matched cohort.

\section{Abstract 043 Table 2}

\begin{tabular}{lccc}
\hline & Pre-accutrak (46) & Post-accutrak (35) & p Value \\
\hline LBBB (n) & 6 & 3 & 0.73 \\
RBBB (n) & 1 & 2 & 0.58 \\
$\begin{array}{l}\text { Mean aortic annulus } \\
\text { size (mm) }\end{array}$ & 22.5 & 22.4 & - \\
$\begin{array}{l}\text { Mean pre-dilation balloon } \\
\text { valvuloplasty size (mm) }\end{array}$ & 23.4 & 23.2 & - \\
$\begin{array}{l}\text { CoreValve size 26 mm } \\
\text { CoreValve size 29 mm }\end{array}$ & 22 & 14 & - \\
$\begin{array}{l}\text { Post-dilation balloon } \\
\text { valvuloplasty performed (n) }\end{array}$ & 12 & 21 & - \\
$\begin{array}{l}\text { New Permanent } \\
\text { pacemaker required }\end{array}$ & 5 & 3 & 0.08 \\
post TAV* (n) & & 3 & 1.0 \\
\hline
\end{tabular}

*New pacemaker post TAVI within 30 days of procedure.

Conclusion The pacing rate was $9.8 \%$ post CoreValve TAVI, which is significantly lower compared to previous estimates. The reduction in pacing rate was due to a successful high deployment strategy and was independent of the Accutrak delivery system. Although the Accutrak catheter has modified CoreValve deployment, it appears to have had little impact on the pacing rate in our cohort. Further evaluation is required to establish whether the Accutrak delivery catheter facilitates a lower pacing rate in middle to low CoreValve implanting centres. We advocate a high CoreValve deployment strategy to ensure a lower pacing rate.

\section{ENDOVASCULAR AND TRANSCATHETER MANAGEMENT OF COARCTATION-RELATED ANEURYSMS IN ADULTS}

doi:10.1136/heartjnl-2012-301877b.44

${ }^{1} \mathrm{~A}$ Khavandi, ${ }^{*} \mathrm{M}$ Hamilton, ${ }^{1} \mathrm{R}$ Martin, ${ }^{1} \mathrm{~A}$ Parry, ${ }^{2} \mathrm{M}$ Brooks, ${ }^{1} \mathrm{~A}$ Baumbach, ${ }^{1} \mathrm{M}$ Turner. ${ }^{1}$ Bristol Heart Institute, UK; ${ }^{2}$ Bristol Royal Infirmary, UK

Introduction Aortic coarctation surgery is associated with a late risk of aneurysm formation. This is particularly seen with patch aortoplasty repair. These aneurysms have a high risk of rupture in the long term as they are frequently false aneurysms. Redo surgery carries significant morbidity and mortality. We describe our experience in treating congenital patients with coarctation-related aortic lesions via endovascular stent grafting and complimentary transcatheter techniques.

Methods Between September 2006 and February 2011, 14 patients with congenital lesions had endovascular or transcatheter descending aortic interventions. 13 received stent grafting with the
Medtronic Valiant system with two of these receiving associated vascular closure with the Amplatzer Vascular Plug (AVP 2). One patient had aneurysm closure with the combination of an Amplatzer septal occluder device and vascular occlusion coils. Data regarding these cases has been retrospectively analysed and described.

Results Fourteen patients were treated for late aneurysm formation following prior coarctation surgery (Patch aortoplasty $n=10$, end-to end anastomosis $n=2$, interrupted arch type with arch or subclavian to descending aortic Dacron bypass $n=2$; mean interval from index surgery 30 years (range 22-38 years)). Mean age at intervention was 40 (range 26-62 years). Two patients were treated as emergencies with haemoptysis secondary to fistulae and haemodynamic instability. One patient presented with pain and another with hoarse voice. All patients had successful treatments of the lesion without the need for reintervention. Mean follow-up is 24 months (range 2 months to 54 months) with no mortality and no significant endoleak. Four patients had non-life threatening complications (exercise left arm ischaemic symptoms requiring late vascular bypass, infected surgical vascular access site requiring re-operation, wound infection and re-exploration of neck for chyle leak-related to elective subclavian artery bypass and ligation of aneursmal subclavian prior to stent graft).

Conclusion Even in complex lesions, endovascular stent grafting with complimentary transcatheter and hybrid techniques provides an excellent option for congenital patients avoiding the risks of repeat surgery and the recovery from redo thoracotomy. Concern about the long-term performance of these grafts in young people would appear to be the only downside of this therapy.

\section{SOCIO-ECONOMIC STATUS AND OUTCOME AFTER PERCUTAENOUS CORONARY INTERVENTION}

doi:10.1136/heartjnl-2012-301877b.45

D A Jones,* S Gallagher, K S Rathod, A K Jain, A Mathur, A Wragg. Barts and the London NHS trust, UK

Introduction The effect of socio-economic status upon outcome after PCI is poorly defined. Limited data exists suggesting an association between social deprivation and worse outcomes following both CABG and myocardial infarction. We sought to determine the effects of socio-economic status (SES) upon long-term outcome after percutaneous coronary intervention (PCI).

Method This was an observational registry consisting of 13770 consecutive patients undergoing PCI at a single centre (January 2005 to August 2011). SES was assigned to each patient according to postcode and ranked according to the corresponding British Index of Multiple Deprivation (IMD) score, which comprises five deprivation quintiles (Q1, least deprived; O5, most deprived). The primary outcome was all cause mortality data obtained from the Office of National Statistics via the BCIS/CCAD national audit. Follow-up was for a median of 3.7 years (IOR 2.0-5.1 years).

Results Patients were predominantly male (74.0\%) and Caucasian $(71.7 \%)$, with a mean ( \pm SD) age of $63.8 \pm 12.0$ years. Median IMD score was 24.4 (13.4 to 38.4), and the total score for each IMD quintile is shown in Abstract 045 table 1. The characteristics of the study population across the five IMD quintiles are also shown in Abstract 045 table 1. Patients in quintile 5 (most deprived) were younger, more likely to be of Asian descent, to be current smokers and had higher rates of previous MI, previous PCI, diabetes mellitus and renal failure. They were also more likely to present as an acute coronary syndrome and have either moderate or poor ejection fraction. Kaplan-Meier estimates of all cause mortality showed increasing rates of long-term mortality for each increase in IMD score quintile, with patients in quintile 5 demonstrating significantly 